\title{
Characterization of drug-release kinetics in trabecular bone from titania nanotube implants
}

This article was published in the following Dove Press journal:

International Journal of Nanomedicine

II September 2012

Number of times this article has been viewed

\section{Moom Sinn Aw' \\ Kamarul A Khalid ${ }^{2,3}$ \\ Karan Gulati' \\ Gerald J Atkins ${ }^{2}$ \\ Peter Pivonka ${ }^{4}$ \\ David M Findlay² \\ Dusan Losic'}

'School of Chemical Engineering, ${ }^{2}$ Discipline of Orthopaedics and

Trauma, The University of Adelaide, Adelaide, SA, Australia; ${ }^{3}$ Department of Orthopaedics, Traumatology and Rehabilitation, Faculty of Medicine, International Islamic University Malaysia, Kuantan, Pahang, Malaysia; ${ }^{4}$ Engineering Computational Biology Group, School of Computer Science and Software Engineering, The University of Western Australia, Perth, WA, Australia
Correspondence: Dusan Losic School of Chemical Engineering, The University of Adelaide, Adelaide, SA 5005, Australia

Tel $+6 \mid 883026862$

Fax +6I 883023683

Email dusan.losic@adelaide.edu.au

David M Findlay

Level 4, Bice Building

Royal Adelaide Hospital

The University of Adelaide

SA 5005, Australia

Tel +6I $88222562 \mid$

Fax +61882323065

Email david.findlay@adelaide.edu.au
Purpose: The aim of this study was to investigate the application of the three-dimensional bone bioreactor for studying drug-release kinetics and distribution of drugs in the ex vivo cancellous bone environment, and to demonstrate the application of nanoengineered titanium (Ti) wires generated with titania nanotube (TNT) arrays as drug-releasing implants for local drug delivery

Methods: Nanoengineered Ti wires covered with a layer of TNT arrays implanted in bone were used as a drug-releasing implant. Viable bovine trabecular bone was used as the ex vivo bone substrate embedded with the implants and placed in the bone reactor. A hydrophilic fluorescent dye (rhodamine B) was used as the model drug, loaded inside the TNT-Ti implants, to monitor drug release and transport in trabecular bone. The distribution of released model drug in the bone was monitored throughout the bone structure, and concentration profiles at different vertical $(0-5 \mathrm{~mm})$ and horizontal $(0-10 \mathrm{~mm})$ distances from the implant surface were obtained at a range of release times from 1 hour to 5 days.

Results: Scanning electron microscopy confirmed that well-ordered, vertically aligned nanotube arrays were formed on the surface of prepared TNT-Ti wires. Thermogravimetric analysis proved loading of the model drug and fluorescence spectroscopy was used to show drug-release characteristics in-vitro. The drug release from implants inserted into bone ex vivo showed a consistent gradual release of model drug from the TNT-Ti implants, with a characteristic threedimensional distribution into the surrounding bone, over a period of 5 days. The parameters including the flow rate of bone culture medium, differences in trabecular microarchitecture between bone samples, and mechanical loading were found to have the most significant influence on drug distribution in the bone.

Conclusion: These results demonstrate the utility of the Zetos ${ }^{\top M}$ system for ex vivo drugrelease studies in bone, which can be applied to optimize the delivery of specific therapies and to assist in the design of new drug delivery systems. This method has the potential to provide new knowledge to understand drug distribution in the bone environment and to considerably improve existing technologies for local administration in bone, including solving some critical problems in bone therapy and orthopedic implants.

Keywords: local drug delivery, Zetos bone bioreactor, drug-releasing implant, drug diffusion

\section{Introduction}

Bone diseases are a major health problem worldwide. They have a highly deleterious effect on both quality of life for patients and health expenditure, representing at least $10 \%$ of annual health care expenditure in many developed countries. ${ }^{1}$ A number of therapeutic approaches have been developed to treat bone diseases, and these 
are dominated by the use of systemic drug administration. However, conventional systemic drug therapies have many limitations, both in general and in bone, such as low efficacy, poor bioavailability and biodistribution, lack of selectivity, and drug overdose and toxicity in nontarget tissues. ${ }^{2,3}$ To overcome these limitations, increase drug effectiveness, and reduce systemic side effects of drugs, localized delivery of therapeutics to bone has been recognized as a promising alternative. ${ }^{4,5}$ Local drug delivery offers many potential advantages, leaving healthy cells or adjacent tissues at other sites unaffected and avoiding serious side effects, providing locally optimal concentrations of often expensive drugs without diluting them across the entire body, and optimizing bioavailability, without rapid breakdown and clearance of drugs, particularly through the liver uptake. ${ }^{6}$

The concept of a skeletal drug delivery system was first introduced by Buchholz et $\mathrm{al}^{7}$ in the 1970s and has been extensively explored since. The development of biocompatible drug-releasing materials and appropriate models to study drug release from these systems, and their effectiveness in bone prior to in vivo study, are recognized as critical issues to be addressed. ${ }^{8,9}$ Numerous biomaterials, either natural or synthetic, and either biodegradable or biologically inert, such as polymethyl methacrylate, poly(lactic-co-glycolic acid), collagen, hyaluronan, chitosan, fibrin, silk, hydroxyapatite, ceramics and injectable calcium phosphate cements, in the form of membranes, granules, hydrogels, matrices, coatings, fibers, sponges, and foams, have been explored in recent years as implants for the delivery of bone active agents. ${ }^{10-15}$ These materials are mostly amorphous, with a large variation of porosity and nonreproducible preparation, which in turn makes the bone therapy nonreproducible. ${ }^{15}$ In most cases, these implants are designed for the delivery of specific drugs and do not have the flexibility to be applied to a wide range of therapeutic agents, such as water-insoluble drugs, drug carriers, or labile agents (proteins and genes). To address these disadvantages, several new nanosynthetic and surface-engineered approaches have been applied for the development of advanced drug-releasing materials and devices with controllable pore size, porosity, and surface functionality at the nanoscale level. ${ }^{5,6,16}$ Among them, titania nanotube (TNT) arrays, generated on titanium (Ti) surfaces by self-ordered electrochemical anodization, are recognized as a particularly promising solution. ${ }^{17,18}$ TNTs have been proven to have many favorable features, including biocompatibility, excellent integration with bone tissue, an ability to promote the growth of hydroxyapatite, high surface area, controllable pore dimensions, surface chemistry, chemical stability, and mechanical rigidity. ${ }^{18-21}$
The second issue of local drug delivery in bone is that most studies of drug release relevant to bone therapies have been performed using in vitro conditions, where the drugloaded implant is interfaced with the solution (buffer) or with cultured bone cells. However, bone is a complex porous material consisting of a solid bone matrix and pore spaces. Two types of bone can be distinguished: compact (or cortical) bone with small pore spaces (Haversian canals) and spongy (or cancellous) bone with large pore spaces filled with bone marrow. The three major types of bone cells are osteoblasts, osteoclasts, and osteocytes. ${ }^{22}$ It is not known how drug release in solution relates to drug release in bone tissues, given that cancellous bone is a highly hydrated tissue, with abundant interstitial fluid surrounded by marrow with a high fat content. ${ }^{5,23}$ Hence, in order to evaluate the potential of local drug delivery systems, the authors sought a suitable system. Because studies in live bone tissue in situ are technically difficult and expensive, the use of an ex vivo bone bioreactor, consisting of a trabecular bone explant, was explored as a suitable alternative. Recently, several three-dimensional (3D) bone reactors have been developed and explored to study the biological aspect of bone cell behavior and bioengineering of bone tissues, including the Zetos system. ${ }^{23-25}$ The Zetos system, devised by David Jones (Department of Experimental Orthopaedics and Biomechanics, Philipps University of Marburg, Marburg, Germany) and Everett Smith (Medical Sciences Center, Madison, WI), enables discs of cancellous bone to be maintained for at least 3 weeks by continuous perfusion with culture medium and daily loading with physiologically relevant strains. ${ }^{24-26}$

The aim of this study was to assess the Zetos bone bioreactor for ex vivo study of drug distribution in bone and to demonstrate the application of nanoengineered $\mathrm{Ti}$ wires generated with TNT arrays (TNT-Ti wires) as drug-releasing implants for local drug delivery (Figure 1A and B). The TNT layer is composed of an array of highly ordered, vertically aligned nanotubes with the capacity to accommodate and release a considerable amount of drug. ${ }^{18-20}$ In previous work, the authors have developed TNT-Ti wires with advanced properties to provide extended and sustained drug release with zero-order kinetics, multidrug delivery with sequential or delayed release, and stimuli-responsive release of drugs using a magnetic field. ${ }^{27-31}$ In the present study, the TNT-Ti wires were loaded with the fluorescence dye (rhodamine B [RhB]) as a model drug and inserted into the middle of trabecular bone discs (Figure 1C), which were placed inside the bone bioreactor with continuous perfusion of culture medium (Figure 1D). The Xenogen IVIS ${ }^{\circledR} 100$ BioPhotonic Imaging $^{\circledR}$ 


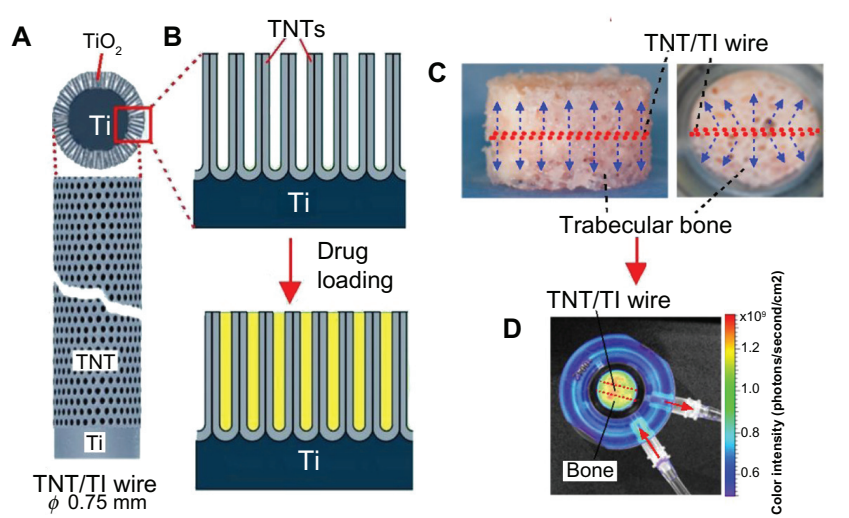

Figure I (A) Diagram of a titanium (Ti) wire with titania nanotube (TNT) arrays on the surface as a drug-releasing implant; (B) diagram of the TNT arrays formed by anodization of the Ti wire and the drug loading inside these nanotubes; (C) trabecular bone with the embedded TNT-Ti implant indicated in the center of the bone core (side and top views); (D) bioluminescence image of the bone core and the embedded TNT-Ti implant inside the perfusion chamber - the distribution of released drug inside the bone is also shown.

Abbreviation: $\mathrm{TiO}_{2}$, titanium dioxide.

system (Caliper Life Sciences, Inc, Hopkinton, MA) was used to monitor in vivo drug release and distribution inside the bone matrix over time (from 1 hour to 5 days). The purpose was to demonstrate the capacity of the Xenogen system to provide in situ measurements of drug release from the implant and to determine the 3D spatial distribution of drugs in bone, the drug-release rate, and drug-release kinetics.

\section{Materials and methods Materials}

Alfa Aesar (Ward Hill, MA) supplied the Ti (99.99\%) wires (diameter $0.75 \mathrm{~mm}$ ). Ethylene glycol, acetone, ammonium fluoride, and $\mathrm{RhB}\left(\mathrm{C}_{28} \mathrm{H}_{31} \mathrm{ClN}_{2} \mathrm{O}_{3}\right.$, with $97 \%$ dye content) were obtained from Sigma-Aldrich Pty Ltd (Sydney, Australia) and used without further purification. High-purity, ultra-grade Milli- ${ }^{\circledR}$ water $(18.2 \mathrm{M} \Omega \mathrm{cm}$ resistivity) (EMD Millipore Corporation, Billerica, MA) with additional filtration $(0.22 \mu \mathrm{m})$ was used for the preparation of all reagents.

\section{Preparation of TNT-Ti wires as drug-releasing implants}

Ti wires were cut to size (approximately $10 \mathrm{~mm}$ in length), polished, cleansed ultrasonically with acetone, rinsed thoroughly with deionized Milli-Q water, and air-dried. TNT layers were then prepared by a two-step electrochemical anodization of $\mathrm{Ti}$ wires in ammonium fluoride/ethylene glycol electrolyte (3\% water and $0.3 \%$ ammonium fluoride) at $20^{\circ} \mathrm{C}$, using a constant voltage of $100 \mathrm{~V}$ for 1 hour, as described previously. ${ }^{32-34}$ Both pore diameter and length of the TNTs were determined by selecting the appropriate voltage $(100 \mathrm{~V})$ and anodization time (1 hour).

\section{Structural characterization of prepared TNT-Ti wires}

Structural characterization of the prepared TNT-Ti wires was performed before and after drug loading and drugrelease experiments in bone using a field emission scanning electron microscope (SEM) (Philips XL 30; Philips Research Eindhoven, Eindhoven, The Netherlands). The samples were cut into small pieces, (approximately $5 \mathrm{~mm}$ in length) mounted on a holder with double-sided conductive tape, and coated with a layer of platinum 3-5 nm thick. Images, with a range of scan sizes at normal incidence and at a $30^{\circ}$ angle, were acquired from the top surface, the bottom surface, and cross-sections.

\section{Loading of the model drug}

$\mathrm{RhB}$, the model drug in this study, was dissolved in water $(50 \mathrm{mg} / \mathrm{mL})$ and used for loading into the TNT-Ti wires. TNT-Ti wires cut into $8 \mathrm{~mm}$ lengths were fully immersed in the $\mathrm{RhB}$ solution to ensure drugs were entirely loaded inside the nanotubes. The wires were rotated every $2-4$ hours during $\mathrm{RhB}$ deposition. After 1-3 days of loading, the wires were removed, dried in air, and then kept under vacuum for 2 hours. Finally, to remove the excess of loaded drug from the TNT surfaces, samples were gently cleaned with a soft tissue after wetting with a small amount of phosphate buffered saline ( $\mathrm{pH}$ 7.2). The wires were sterilized using low-temperature hydrogen peroxide gas plasma ( Sterrad $^{\circledR} 100 \mathrm{NX}^{\mathrm{TM}}$ System, Advanced Sterilization Products (ASP),Division of Ethicon Inc, a Johnson \& Johnson company, Irvine, CA).

\section{Quantification of drug loading}

To quantify the amount of RhB loaded into the TNT-Ti wires, thermogravimetric analysis (TGA), which measures the mass change (weight loss) in a sample as a function of temperature, was performed using a Hi-Res Modulated TGA 2950 (TA Instruments, New Castle, DE). The drug-loaded TNT-Ti wire was mounted on the platinum pan and heated from room temperature (at $20^{\circ} \mathrm{C}$ ) to $800^{\circ} \mathrm{C}$ in a furnace at a scanning rate of $10^{\circ} \mathrm{C}$ per minute under a nitrogen gas flow of $50 \mathrm{~mL}$ per minute. To find the correct decomposition range and peak of $\mathrm{RhB}$ model drugs, TGA of the pure RhB was performed in separate experiments. A thermogram showing a decrease in weight was identified from the TGA software (Q Series Thermal Analysis, Universal Analysis 2000; TA Instruments) and was used to calculate the loaded amount of RhB. 


\section{Preparation of bovine trabecular bone cores}

The sternum of a 16-month-old steer was harvested from the freshly slaughtered animal and kept in cold sterile saline $(0.85 \%)$ prior to processing. All soft tissues were removed from the sternum, which was then manually cut into sagittal sections using a hacksaw. Care was taken to maintain the sterility and viability of the samples at all times. Prepared sections were kept immersed in a prewash medium, consisting of high-glucose Dulbecco's Modified Eagle Medium (Life Technologies Corporation, Carlsbad, CA), with $20 \mathrm{mM}$ of 4-(2-hydroxyethyl)-piperazineethanesulfonic acid, $2.4 \mathrm{mg} / \mathrm{mL}$ of benzylpenicillin, $3.2 \mathrm{mg} / \mathrm{mL}$ of gentamicin sulfate, and $4 \mu \mathrm{g} / \mathrm{mL}$ of amphotericin B. Bone cylinders, $10 \mathrm{~mm}$ in diameter, were prepared from the bone sections using an industrial drilling machine (Model G0517 Mill/Drill, Grizzly Industrial ${ }^{\circledR}$, Inc, Muncy, PA) and a custom-made diamond drill bit (Figure 2A). Bone samples were continually immersed in cold sterile saline $(0.85 \%)$ on a custom-made polyoxymethylene drilling jig to prevent desiccation and/or thermal necrosis. The bone cylinders were mounted onto a custommade platform and milled to a $5 \mathrm{~mm}$ thickness using a $10 \mathrm{~mm}$ diameter tungsten carbide bit, with the bone pieces immersed throughout in cold sterile saline $(0.85 \%)$. The resulting bone cores consisted of uniform trabecular bone without any visible cartilage. All bone cores had the marrow removed by
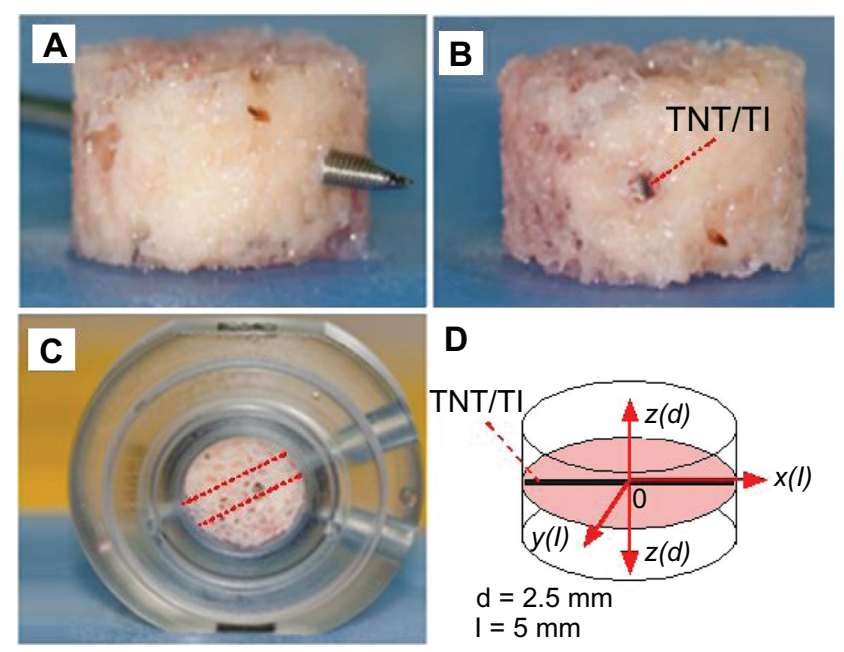

D

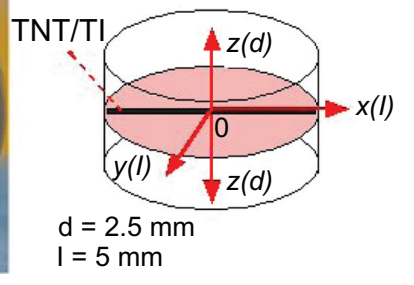

Figure 2 Steps for preparation of bovine trabecular bone cores for ex vivo studies using the Zetos three-dimensional bone bioreactor: (A) bone core with marrow removed and a hole in the center drilled with a surgical Kirschner wire; (B) drug-releasing implant, based on a titanium (Ti) wire with titania nanotube (TNT) arrays on the surface, embedded in the center of the bone; (C) the bone core with implant placed inside the chamber with the same orientation (angle) to the flow of prelusion medium into the bone; (D) diagram of bone core showing inserted implant in the middle and the directions (with different axes) used to monitor drug concentration.

Note: In this work, only drug distribution from the central point of the implant (0) at the bottom vertical plane $(x-z)$ and the horizontal plane $(x-y)$ is presented. washing under pressure using a dental water jet (WP-450A; Water Pik, Inc, Fort Collins, CO). The bone cores were stored in the prewash medium at $4^{\circ} \mathrm{C}$ prior to use.

\section{In vitro drug release from $\mathrm{TNT}-\mathrm{Ti}$ implants}

The RhB-loaded TNT-Ti wires were immersed in $5 \mathrm{~mL}$ of phosphate buffered saline ( $\mathrm{pH} 7.2)$ at room temperature and an in vitro drug-release study was performed, using a procedure described previously. ${ }^{27}$ Briefly, aliquots of buffer solution were analyzed every 5-15 minutes and then twice daily until the release reached completion. The aliquots were placed in a quartz cuvette and the concentration of released RhB was measured using a Cary Eclipse fluorescence spectrophotometer (Varian, Inc, Palo Alto, CA) with excitation and emission wavelengths of 510 and $625 \mathrm{~nm}$, respectively.

\section{Insertion of TNT-Ti wires into the bovine bone cores}

A hole was drilled through each bone core using a sterilized $1.1 \mathrm{~mm}$ diameter Kirschner wire (surgical grade stainless steel sharp pin) (Figure 2A). A TNT-Ti wire was carefully inserted into the hole, fitting tightly into the center of the bone core (Figure 2B), and the bone was secured in the custom-made culture chamber, as shown in Figure 2C. The orientation of the implants inside the bones connected to the inlets and outlets of the bioreactor was kept the same for all samples (Figure 2C). Bone cores were then loaded into the sterile perfusion chambers that were compatible with the Zetos ${ }^{\mathrm{TM}}$ loading system (Figure 3 ). The study was performed with and without different mechanical loading, but only results without loading have been presented, because of data congestion.

\section{Ex vivo drug release in trabecular bone}

Six trabecular bone cores, four with TNT-Ti wires loaded with $\mathrm{RhB}$ and two with TNT-Ti wires without $\mathrm{RhB}$, were prepared and perfused with culture medium during the course of this study. The study was performed in triplicate. The 3D bone bioreactor comprises a set of cross-flow culture chambers, allowing a constant perfusion of bone samples with culture medium at a rate of $7 \mathrm{~mL}$ per hour (Figure 3). The entire apparatus was maintained at $37^{\circ} \mathrm{C}$. The color intensity of the dye from the TNT-Ti wires and into the bone core was measured at 1, 4, and 24 hours and 4 and 5 days (Figure 1D) using the Xenogen IVIS 100 (In vivo imaging system). This measurement provided an accurate estimate of the amount of dye released, as this amount is directly proportional to 


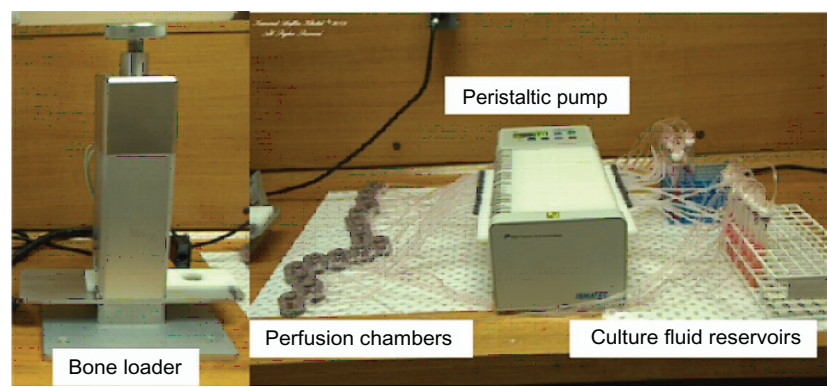

Figure 3 The Zetos three-dimensional bone bioreactor (devised by David Jones [Department of Experimental Orthopaedics and Biomechanics, Philipps University of Marburg, Marburg, Germany] and Everett Smith [University of Wisconsin, WI]) used for the ex vivo study of drug distribution inside trabecular bone.

the color intensity (photons/second/cm2). The luminescence imaging mode was set at an exposure time of 1 minute, with medium-size binning, open emission filter, and $25 \mathrm{~cm}$ view field. The dimension of the pixel count was $2 \times 2$ (width by height), with a subject area of $0.76 \mathrm{~cm}^{2}$. The photon count rate per unit area, based on the control samples with dye and no dye, was used as the benchmark for the diffusion measurement in terms of the time and spatial distance across the bone. The drug concentration values from the collected images were obtained by calibration, using the known concentration of the model drug on the TNT-Ti wire surface and the control wire with no drug. The parameter mass flux $\left(\mathrm{mg} / \mathrm{cm}^{2}\right.$. second) was calculated from the number of photons collected as the $\mathrm{RhB}$ dye passed through a unit area by a linear conversion of units. Molar flux ( $\mathrm{mol} / \mathrm{cm}^{2}$. second), mass flow ( $\mathrm{mg} / \mathrm{second}$ ), and volumetric flow rate $(\mathrm{mL} / \mathrm{second})$ can also be derived from the primary data available from the results. From these images, the values of drug concentration in bone at different distances along the $x, y$, and $z$ axes from the center of the bone (position of the implant) over time (from 1 hour to 5 days) were obtained. These results can be used to create 3D drug distribution graphs, but in this study only two-dimensional concentration profiles in the horizontal and vertical directions from the TNT-Ti implant surface are presented to demonstrate the capability of the system.

\section{Results and discussion Structural characterization of TNT-Ti wires}

The structure of prepared TNT-Ti wires was characterized by SEM and the typical morphology of the wires is summarized in Figure 4. Figure 4A shows the whole TNT-Ti wire $(12 \mathrm{~mm})$ (inset) and a low-resolution SEM image confirming the growth of a TNT film on the curved surface of the Ti wire. The SEM image shows randomly dispersed vertical
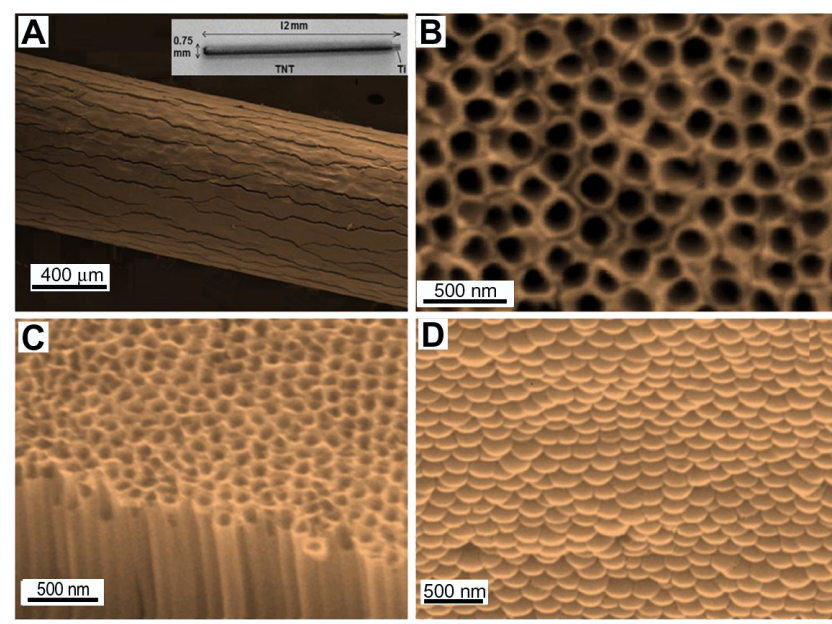

Figure 4 Scanning electron microscope images of (A) a drug-releasing implant based on a titanium (Ti) wire with titania nanotube (TNT) arrays on the surface (whole wire shown in inset), (B) the top surface of the TNTs, $(\mathbf{C})$ a cross-sectional view showing hollow nanotube structures, and (D) the bottom surface, showing closed ends of the nanotube structures (the TNT layer was removed from the underlying Ti for imaging purposes).

cracks across the entire length of the wire, caused by the radial growth of TNTs on the curved surface and mechanical stress through volume expansion. These cracks have not been observed on TNT films grown on planar Ti surfaces. However, the generation of TNTs on wire did not significantly change the original mechanical stability and adherence of the TNT layer on Ti. High-resolution SEM images of the top surface, cross-section, and bottom surface of the TNT layer show a vertically aligned and densely packed array of uniform nanotubes, with diameters of $140 \pm 10 \mathrm{~nm}$ and lengths of $50 \pm 2 \mu \mathrm{m}$, across the entire structure (Figure 4B-D). TNTTi wires occupy a very small amount of space and are easy to implant into bone and be removed. These features show many advantages for implantable orthopedic applications in comparison with existing commercial bone implants such as polymer gels and bone cements.

\section{The in vitro drug-release characterization}

TGA was performed to determine the amount of drug loaded into the nanotubular structures of the TNT-Ti wires. A total $\mathrm{RhB}$ loading of $0.27 \mathrm{mg}$ was found per $8 \mathrm{~mm}$ length of TNT-Ti wire. However, the drug loading of TNT-Ti wires can be optimized by selecting appropriate TNT dimensions and applying surface modifications. ${ }^{35}$ Thus, it is possible to customize TNT-Ti platforms to meet specific requirements for implantable drug delivery in bone, depending on the required dosage, properties of drugs, and proposed bone therapy (infections, bone cancer, and so forth). 
In vitro drug release of the model drug $(\mathrm{RhB})$ loaded into the TNT-Ti wires in buffer solution is presented in Figure 5. The drug-release kinetics can be described in two phases, the first phase showing an initial burst release during the first 6 hours, followed by the second phase with a slow release of the remaining drug. The initial burst denoted by the straight portion of the curve, with a release of about $65 \%$ in buffer solution, is regarded as a first-order release. The fast initial release accounts for the fast diffusion of the drug molecules physisorbed on the top and upper parts of the TNTs. In the second phase, drug release from the TNT-Ti implant was very slow and underwent a linearly increasing cumulative release over a period of 3 days. The release mechanism of this phase is controlled by diffusion from the long nanotube structures. ${ }^{36,37}$ The best-fitting model for this phase was obtained using the Higuchi equation followed by the zeroorder release, which describe drug release from an insoluble matrix. ${ }^{38}$ The square root of a time-dependent process is based on the Fickian diffusion law, where the diffusion-controlled release rate of drug molecules decreases as a function of time due to a reduction in concentration gradient. The pharmaceutical dosage following a zero-ordered pattern is the ideal profile of drug release because it provides the same amount of drug elution per unit of time. ${ }^{30}$ These results confirmed previous reports showing suitable drug-releasing characteristics of TNT-Ti implants as drug-releasing platforms for local drug delivery applications. ${ }^{39,40}$ In the following section, the authors investigate the performance of the TNT-Ti implants when implanted in trabecular bone in a bioreactor environment.

\section{The characterization of drug diffusion in trabecular bone ex vivo}

The structure of bone is complex and is expected to have a significant impact on both drug-release kinetics and drug

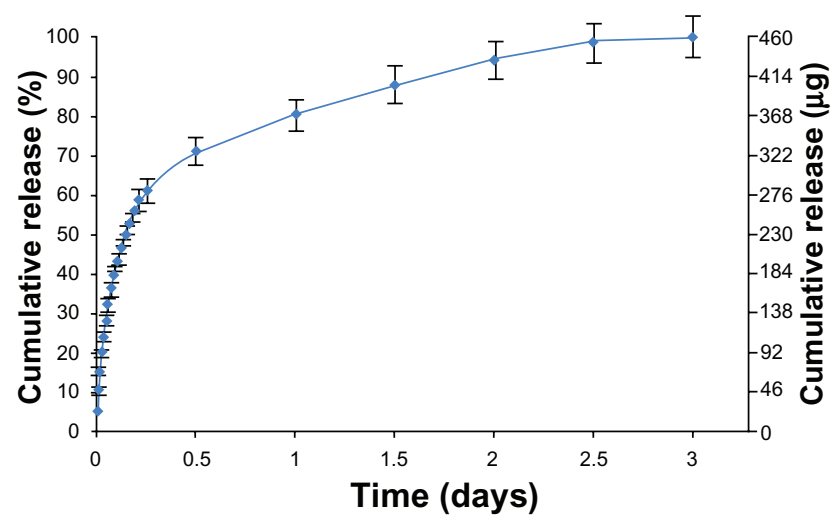

Figure 5 In vitro drug release from implants (based on titanium wires with titania nanotube arrays on their surfaces) into phosphate buffer. distribution in bone. Bioluminescence images created at different times (ie, 1, 4, 24, and 120 hours) for the 5-day experiment on drug-release studies using the Xenogen IVIS (in vivo imaging system) are presented in Figure 6. Results clearly show an increasing concentration of the model drug within the bone model. To demonstrate drug release in the $3 \mathrm{D}$ bone matrix, a series of drug concentration profiles were collected from these images. These profiles show the changes of drug concentration in bones across all directions $(x, y$, and $z$ axes) from the TNT-Ti implant. To simplify the presentation of results, only two-dimensional concentration profiles of the drug in vertical $(x-z)$ and horizontal $(x-y)$ directions from the implant are presented in this work (Figure 7). The graphs show significant changes in $\mathrm{RhB}$ concentration in bone over time, confirming that both processes, ie, the diffusion of RhB from the TNT-Ti implants and the diffusion of RhB into bone, occurred. Initially, the release of $\mathrm{RhB}$ from the TNT-Ti implants followed burst release kinetics (Figure 5), and the highest concentration of $\mathrm{RhB}$ was observed at locations close to the implant surface, with zero concentration at greater distances from the implant. Over time, as more RhB was released, the concentration increased further from the implant, showing the spread of RhB across the bone tissue in all directions (results for only two directions, vertical and horizontal, are presented). After 4 and 5 days of release, the highest concentration was observed at the outer locations of the bone, which was 3.5-5 mm away from the surface of the implant, showing a concentration of nearly 15-20 $\mu \mathrm{g}$. After 5 days of release, there was no measurable drug remaining in the TNT-Ti wires, as confirmed by TGA, which is consistent
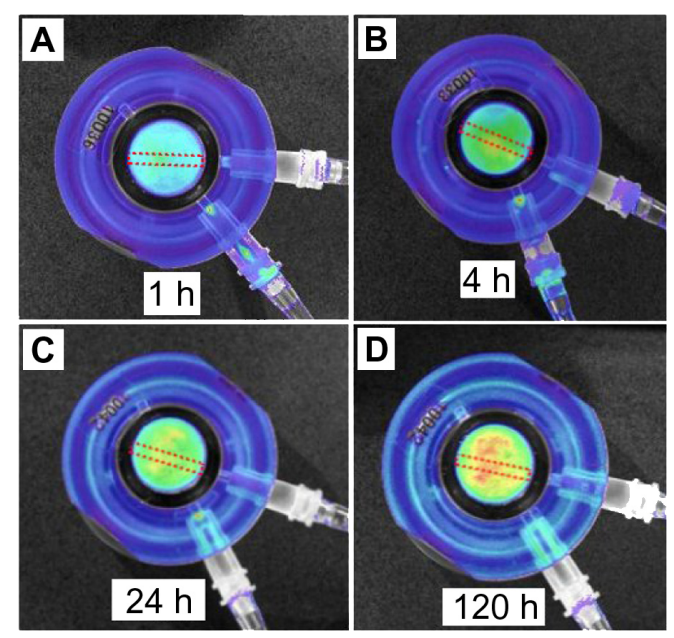

Figure 6 Bioluminescence images of bone with inserted drug-releasing implant (based on a titanium wire with titania nanotube arrays on the surface) for local drug delivery: distribution of released model drug (rhodamine B) taken at (A) I, (B) 4 , (C) 24, and (D) 120 hours (h) using the Xenogen IVIS ${ }^{\circledR} 100$ (Caliper Life Sciences, Inc, Hopkinton, MA) in vivo imaging system. 


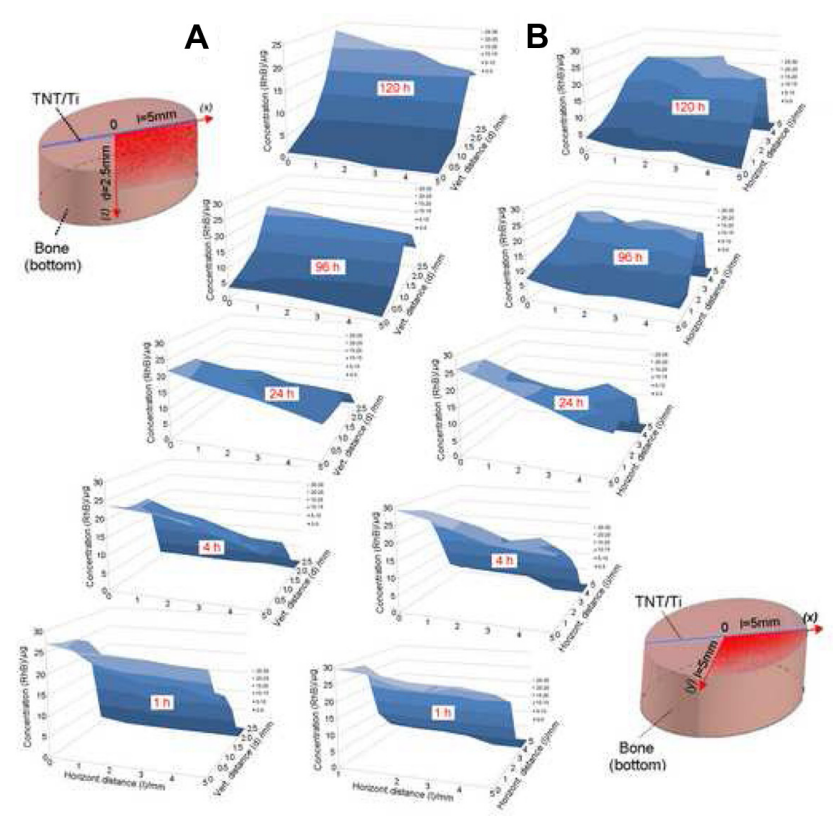

Figure 7 Two-dimensional concentration graphs of the model drug (rhodamine $B[R h B]$ ) released from implants based on titania nanotube (TNT) arrays on the surface of titanium $(\mathrm{Ti})$ wires. The graphs show spatial distribution in the trabecular bone samples: drug distribution is presented at $(\mathbf{A})$ the vertical (vert) plane $(x-z)$ of the bottom part of the bone and (B) the horizontal (horizont) plane $(x-y)$ from the implant surface at different release times (I, 4, 24, 96, and I 20 hours [h]).

Note: The concentration changes are presented only for a selected area of bone (one quadrant), as illustrated in the bone diagrams (left and right).

with the diffusion of $\mathrm{RhB}$ into the bone tissue. The graphs also show that the spatial $\mathrm{RhB}$ distribution in bone is not uniform, which is likely explained by the variable nature of the internal bone microarchitecture and the influence of culture medium flow inside the bone chamber. ${ }^{23}$

\section{The effects of perfusion of culture medium on the drug diffusion pattern}

A continuous flow chamber with culture medium was used in the bone bioreactor to simulate in vivo conditions, mimicking bone fluid flow and maintaining bone viability. It is reasonable to expect that the flow of culture medium at the interface between bone and the TNT-Ti implant will influence the release kinetics of the drug from the TNTs and have a significant impact on drug distribution. To confirm the impact of flow on drug transport in the bone, the authors performed a control experiment without culture flow. The comparative concentration graphs obtained at different distances from the implant at vertical and horizontal directions unequivocally show a difference in diffusion kinetics when external flow is not applied (Figure 8). The drug concentration at both horizontal and vertical directions when perfusion was used was significantly greater than in static conditions, where drug distribution was generated not only by the free diffusion of the drug. This result suggests that the diffusion of drug in bone is a very slow process and should be considered in drug delivery for bone therapy where the access of a drug to bone sites without blood supply is required.

Data analysis of the concentration patterns of RhB in bone from a number of samples showed some unexpected differences and inconsistencies that cannot be linked to the influence of medium flow. The authors assumed these differences were attributed to differences in trabecular microarchitecture between bone samples; addressing this issue is outside the scope of this paper and will be presented in the next publication. When bone samples containing TNT-Ti implants were loaded by applying physiologically relevant mechanical strains, drug diffusion was increased (data not shown); these results will also be presented in a following publication. Therefore, bone loading, together with the presence of the vasculature, is likely to change the drug diffusion kinetics, although in vivo models will be required to fully investigate drug release and diffusion in situ.

\section{The ex vivo study of drug-release kinetics in a 3D bone environment}

Finally, to demonstrate the utility of the Zetos bone bioreactor for studying drug-release kinetics in bones, two examples showing changes of cumulative drug release in bone over time are presented in Figure 9. The graphs show cumulative drug release in bone over a period of time (from 1 hour to 5 days) at three different distances in vertical $(z)$ and horizontal $(x)$ directions from the surface of the TNT-Ti implant. The drug-release kinetics in buffer solution showed significant differences compared with release from TNT-Ti implants in the ex vivo bone. An initial burst release ( 6 hours), with $65 \%$ of the cumulative release and the first-order release kinetics, was observed for in vitro release of RhB into the buffer solution (Figure 5). In comparison, ex vivo release in bone showed considerably lower initial release $(<20 \%)$, without burst release. Drug release over this time was slow, with a continuous and gradual cumulative increase over time. This behavior is likely governed by the diffusion of drug from the TNTs, flow rate of culture medium at the TNT-Ti interface, and flow rate of culture medium across the bone. The release pattern of drug inside the bone follows first-order kinetics in all directions from the implant surface, but some anomalies were shown after 3 days in the vertical direction $(z=0)$. This is possibly the result of the accumulation of RhB at the edge of the bone sample. At this stage, the authors cannot quantitatively explain the drug-release 

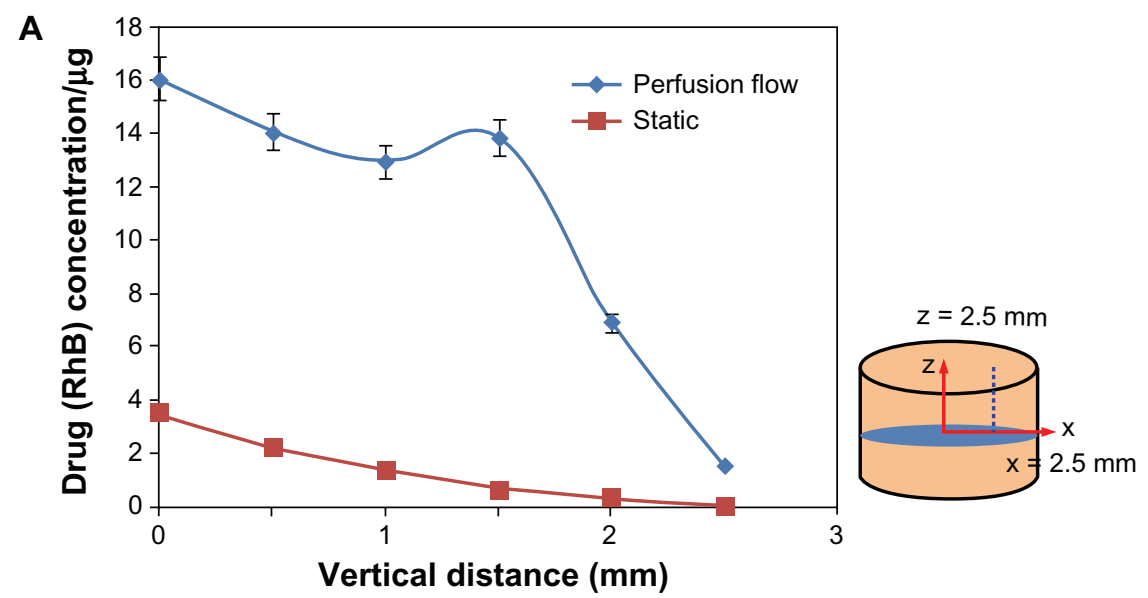

B

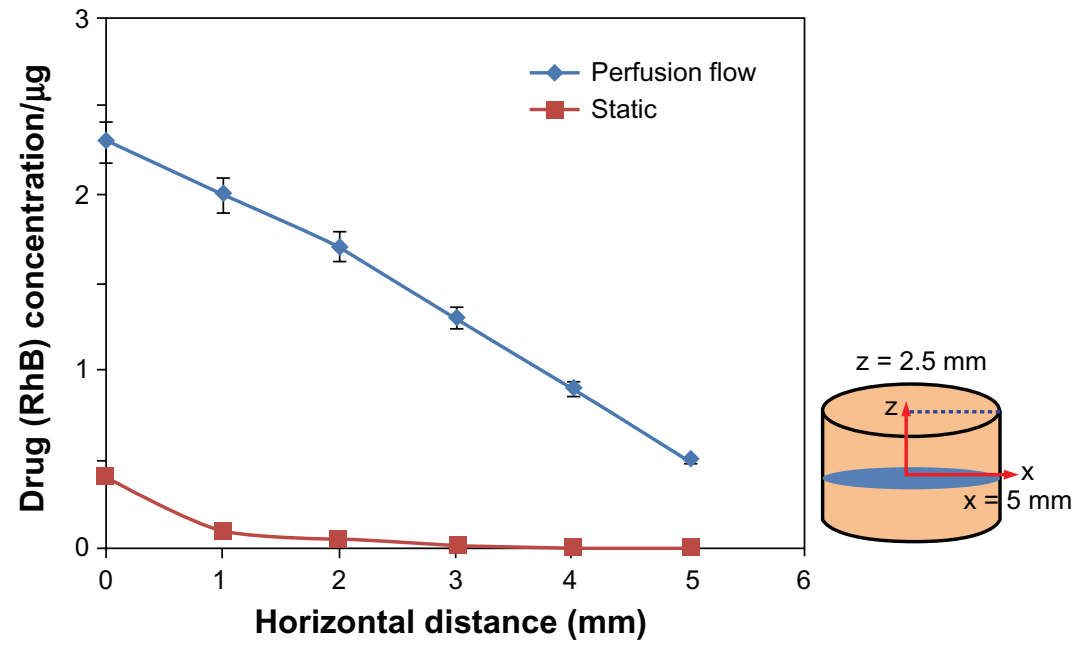

Figure 8 Influence of the physiological solution (culture medium) flow rate in the bone bioreactor on the distribution of the model drug (rhodamine B [RhB]) released from implants based on titania nanotube arrays on the surface of titanium wires. The flow rate of $7 \mathrm{~mL} /$ hour was compared with the static condition (no flow) after 24 hours of drug release. The bone diagrams on the right indicate the locations at $(\mathbf{A})$ horizontal $(5 \mathrm{~mm})$ and $(\mathbf{B})$ vertical distances $(2.5 \mathrm{~mm})$ from the surface of the implant where concentration measurements were taken.

kinetics and transport of the model drug in the trabecular bone samples, owing to the complex interactions of transport mechanisms, including the diffusive and advective components. The importance of this current study is to demonstrate the capability of the ex vivo bone system to generate valuable experimental data. These data can not only be used to define the transport kinetics of drugs in bone but also to optimize drug-release characteristics of bone implants using TNT technology. Future work will include a statistical analysis of repeated experiments, the
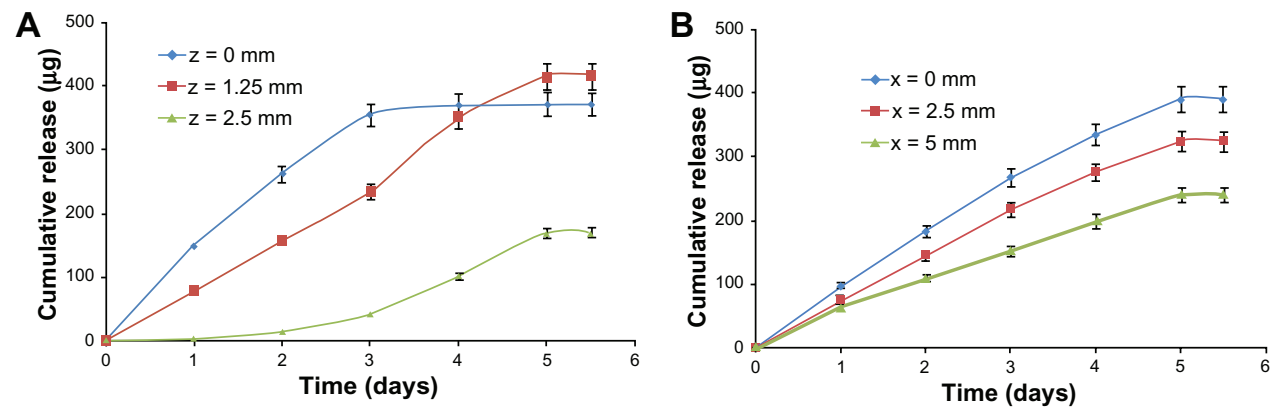

Figure 9 Cumulative release of the model drug (rhodamine B) from drug-releasing implants (based on titania nanotube arrays on the surface of titanium wires) in trabecular bone, showing changes of concentration at different times (from I hour to 5 days). The concentration changes over time are presented for selected locations at (A) vertical $(x-z)$ and $(\mathbf{B})$ horizontal $(x-y)$ distances from the surface of the implant. 
influence of flow rate, mechanical loading, an optimization of the design of the bioreactor.

\section{Conclusion}

In summary, the concept of using a Zetos three-dimensional bone bioreactor as a characterization tool for the ex vivo study of drug-release kinetics and drug distribution in bone was introduced. Viable bovine trabecular bone was used as the ex vivo bone substrate embedded with a drug-releasing implant based on nanoengineered Ti wires covered with a layer of TNT arrays. The utility of the Zetos system for studying drug-release kinetics and transport of drugs in bones is demonstrated for the first time. Successful ex vivo experiments lead to the conclusion that the release pattern and molecular disposition of drugs can be precisely estimated in the bone core with respect to time and location, showing the potential of this technology to predict a real drug concentration and distribution in bone, which is not possible with existing in vitro models. This method has the potential to provide new knowledge to understand drug distribution in complex bone environment and to considerably improve existing technologies for local administration in bone, including solving some critical problems in bone therapy and orthopedic implants. TNT-Ti wire can be considered as a safe drugreleasing implant for localized drug delivery in bone with the potential for clinical application in a range of bone therapies, including those for bone infection, bone inflammation, and bone cancer.

\section{Acknowledgments}

The authors gratefully acknowledge the financial support of the Australian Research Council (DP 120101680) and the National Health and Medical Research Council (ID 565375).

DL receives funding from the Australian Research Council as a Future Fellow (FT110100711). KAK receives funding from the International Islamic University Malaysia and the Malaysian Government.

\section{Disclosure}

The authors report no conflicts of interest in this work.

\section{References}

1. Rodan GA, Martin TJ. Therapeutic approaches to bone diseases. Science. 2000;289(5484):1508-1514.

2. Mainardes RM, Silva LP. Drug delivery systems: past, present, and future. Curr Drug Targets. 2004;5(5):449-455.

3. LaVan DA, McGuire T, Langer R. Small-scale systems for in vivo drug delivery. Nat Biotechnol. 2003;21(10):1184-1191.

4. Jain AK, Panchagnula R. Skeletal drug delivery systems. Int J Pharm. 2000;206(1-2):1-12.
5. Porter JR, Ruckh TT, Popat KC. Bone tissue engineering: a review in bone biomimetics and drug delivery strategies. Biotechnol Prog. 2009; 25(6):1539-1560.

6. Wu P, Grainger DW. Drug/device combinations for local drug therapies and infection prophylaxis. Biomaterials. 2006;27(11):2450-2467.

7. Buchholz HW, Elson RA, Engelbrecht E, Lodenkämper H, Röttger J, Siegel A. Management of deep infection of total hip replacement. JBone Joint Surg Br. 1981;63-B(3):342-353.

8. Soundrapandian C, Sa B, Datta S. Organic-inorganic composites for bone drug delivery. AAPS Pharm Sci Tech. 2009;10(4):1158-1171.

9. Simchi A, Tamjid E, Pishbin F, Boccaccini AR. Recent progress in inorganic and composite coatings with bactericidal capability for orthopaedic applications. Nanomedicine. 2011;7(1):22-39.

10. Zhao L, Chu PK, Zhang Y, Wu Z. Antibacterial coatings on titanium implants. J Biomed Mater Res B Appl Biomater. 2009;91(1):470-480.

11. Tran PA, Sarin L, Hurt RH, Webster TJ. Opportunities for nanotechnology-enabled bioactive bone implants. J Mater Chem. 2009;19:2653-2659.

12. Zilberman M, Elsner JJ. Antibiotic-eluting medical devices for various applications. J Control Release. 2008;130(3):202-215.

13. Lewis G. Alternative acrylic bone cement formulations for cemented arthroplasties: present status, key issues, and future prospects. J Biomed Mater Res B Appl Biomater. 2008;84(2):301-319.

14. Arcos D, López-Noriega A, Ruiz-Hernández E, Terasaki O, Vallet-Regí M. Ordered mesoporous microspheres for bone grafting and drug delivery. Chem Mater. 2009;21(6):1000-1009.

15. Hoppe A, Güldal NS, Boccaccini AR. A review of the biological response to ionic dissolution products from bioactive glasses and glass-ceramics. Biomaterials. 2011;32(11):2757-2774.

16. Liu H, Webster TJ. Nanomedicine for implants: a review of studies and necessary experimental tools. Biomaterials. 2007;28(2):354-369.

17. Ghicov A, Schmuki P. Self-ordering electrochemistry: a review on growth and functionality of $\mathrm{TiO}(2)$ nanotubes and other self-aligned MO(x) structures. Chem Commun (Camb). 2009;(20): 2791-2808.

18. Losic D, Simovic S. Self-ordered nanopore and nanotube platforms for drug delivery applications. Expert Opin Drug Deliv. 2009;6(12): 1363-1381.

19. Roy P, Berger S, Schmuki P. TiO(2) nanotubes: synthesis and applications. Angew Chem Int Ed Engl. 2011;50(13):2904-2939.

20. Popat KC, Eltgroth M, LaTempa TJ, Grimes CA, Desai TA. Titania nanotubes: a novel platform for drug-eluting coatings for medical implants? Small. 2007;3(11):1878-1881.

21. Rani S, Roy SC, Paulose M, et al. Synthesis and applications of electrochemically self-assembled titania nanotube arrays. Phys Chem Chem Phys. 2010;12(12):2780-2800.

22. Sikavitsas VI, Temenoff JS, Mikos AG. Biomaterials and bone mechanotransduction. Biomaterials. 2001;22(19):2581-2593.

23. Davies CM, Jones DB, Stoddart MJ, et al. Mechanically loaded ex vivo bone culture system 'Zetos': systems and culture preparation. Eur Cell Mater. 2006;11:57-75.

24. David V, Guignandon A, Martin A, et al. Ex vivo bone formation in bovine trabecular bone cultured in a dynamic $3 \mathrm{D}$ bioreactor is enhanced by compressive mechanical strain. Tissue Eng Part A. 2008;14(1): $117-126$.

25. Endres S, Kratz M, Wunsch S, Jones DB. Zetos: a culture loading system for trabecular bone. Investigation of different loading signal intensities on bovine bone cylinders. J Musculoskelet Neuronal Interact. 2009;9(3): 173-183.

26. David V, Martin A, Lafage-Proust MH, et al. Mechanical loading down-regulates peroxisome proliferator-activated receptor gamma in bone marrow stromal cells and favors osteoblastogenesis at the expense of adipogenesis. Endocrinology. 2007;148(5):2553-2562.

27. Gulati K, Ramakrishnan S, Aw MS, Atkins GJ, Findlay DM, Losic D. Biocompatible polymer coating of titania nanotube arrays for improved drug elution and osteoblast adhesion. Acta Biomater. 2012;8(1): 449-456. 
28. Simovic S, Losic D, Vasilev K. Controlled drug release from porous materials by plasma polymer deposition. Chem Commun (Camb). 2010; 46(8):1317-1319.

29. Aw MS, Addai-Mensah J, Losic D. A multi-drug delivery system with sequential release using titania nanotube arrays. Chem Commun (Camb). 2012;48(27):3348-3350.

30. Aw MS, Simovic S, Addai-Mensah J, Losic D. Polymeric micelles in porous and nanotubular implants as a new system for extended delivery of poorly soluble drugs. J Mater Chem. 2011;21:7082-7089.

31. Aw MS, Addai-Mensah J, Losic D. Magnetic-responsive delivery of drug-carriers using titania nanotube arrays. J Mater Chem. 2012;22: 6561-6563.

32. Vasilev K, Poh Z, Kant K, Chan J, Michelmore A, Losic D. Tailoring the surface functionalities of titania nanotube arrays. Biomaterials. 2010;31(3):532-540.

33. Kant K, Losic D. A simple approach for synthesis of TiO(2) nanotubes with through-hole morphology. Phys Status Solidi RRL. 2009;3(5): 139-141.
34. Gulati K, Aw MS, Losic D. Drug-eluting Ti wires with titania nanotube arrays for bone fixation and reduced bone infection. Nanoscale Res Lett. 2011;6:571.

35. Popat KC, Leoni L, Grimes CA, Desai TA. Influence of engineered titania nanotubular surfaces on bone cells. Biomaterials. 2007;28(21): 3188-3197.

36. Roy P, Kim D, Lee K, Spiecker E, Schmuki P. TiO(2) nanotubes and their application in dye-sensitized solar cells. Nanoscale. 2010;2(1):45-59.

37. Wang S. Ordered mesoporous materials for drug delivery. Micropor Mesopor Mater. 2009;117(1-2):1-9.

38. Singhvi G, Singh M. Review: in-vitro drug release characterization models. Int J Pharm Studies Res. 2011;2(1):77-84.

39. Alpaslan E, Ercan B, Webster TJ. Anodized $20 \mathrm{~nm}$ diameter nanotubular titanium for improved bladder stent applications. Int J Nanomedicine. 2011;6:219-225.

40. Gulati K, Aw MS, Losic D. Nano-engineered Ti wires for local delivery of chemotherapeutics in brain. Int J Nanomedicine. 2012;7: 2069-2076.
International Journal of Nanomedicine

\section{Publish your work in this journal}

The International Journal of Nanomedicine is an international, peerreviewed journal focusing on the application of nanotechnology in diagnostics, therapeutics, and drug delivery systems throughout the biomedical field. This journal is indexed on PubMed Central, MedLine, CAS, SciSearch $\AA$, Current Contents ${ }^{\circledR} /$ Clinical Medicine,

\section{Dovepress}

Journal Citation Reports/Science Edition, EMBase, Scopus and the Elsevier Bibliographic databases. The manuscript management system is completely online and includes a very quick and fair peer-review system, which is all easy to use. Visit http://www.dovepress.com/ testimonials.php to read real quotes from published authors. 